\title{
Advanced characterization of microbeads replacement from cellulose acetate based on empty fruit bunches and dried jackfruit leaves
}

\author{
Dewi Tristantini ${ }^{*}$, and Andersen Yunan ${ }^{1}$. \\ ${ }^{1}$ Department of Chemical Engineering, Faculty of Engineering, University of Indonesia, Depok, 16424, Indonesia.
}

\begin{abstract}
The existing polymer microbeads for skin extraction ingredients have many disadvantages in environment. The application of cellulose has been proven in the pharmaceutical field in the form of beads on drug release can be a substitute alternative to polymer microbeads that will be prohibited. Based on several criteria and past researches, cellulose acetate meets the criteria for microbeads replacement. Cellulose is available in large quantities in the world, and many studies has proven its application on a wide scope. Empty Fruit Bunches (EFB) and Dried Jackfruit Leaves (DJL) are widely distributed raw materials in Indonesia so that they can be used as a substitute for microbeads. The FTIR and SEM-EDX tests were conducted to determine the functional groups of cellulose acetate and morphology formation of cellulose acetate both raw materials and their chemical composition. In FTIR testing, the typical absorption of EFB and DJL cellulose acetate is produced by $\mathrm{C}=\mathrm{O}$ groups for EFB at wavelength $1721,36 \mathrm{~cm}^{-1}$ and $\mathrm{DJL}$ at wavelength $1725,22 \mathrm{~cm}^{-1}$, whereas at SEM-EDX, DJL cellulose acetate asymmetrical cylinders and rare small pores on the surface and cellulose acetate TKKS cylindrical symmetrical with small pores on its surface. The chemical components of EFB and DJL cellulose acetate exhibit organic elements of carbon $(\mathrm{C})$ and oxygen $(\mathrm{O})$.
\end{abstract}

\section{Introduction}

The existence of polymer microbeads as a scrub material that is widely used in cosmetics and personal care becomes a new problem for the environment. The polymers used as microbeads in cosmetics are very persistent and require a very long time to be degraded naturally in nature. The very small size of the microbeads polymer causes these particles to not be filtered from household filters and end up in the oceans. Based on the facts found, the amount of pollutants that accumulate in microbeads such as PCBs (Polychlorinated biphenyls) reaches 100,000-1,000,000 times the amount detected in seawater (National Oceanic Atmosphere Association, 2011). Marine animals are often wrong in interpreting microbeads particles as their food and consequently microbeads enter into the human food chain when consuming these marine animals.

Based on several criteria, cellulose acetate meets the substitute criteria of microbeads. Cellulose is a biopolymer whose material is available in large quantities in the world, and many studies that prove its application on a wide scope.
The application of cellulose that has been proven in the pharmaceutical field in the form of beads on drug release can be a substitute alternative to polymer microbeads that will be prohibited. In addition, cellulose can be degraded in waste treatment, therefore it will not pollute the marine biota.

The research about basic characteristics from cellulose acetate of EFB and DJL has been done previouly [1]. Past similar research shows that cellulose acetate of EFB and DJL has the basic characteristics, which are density, particle size, water and oil absorption need to be the replacement for microbeads. This study aims to examine advanced physical characteristics, such as Fourier Transformaton Infra Red (FTIR) to know cellulose acetate functional group and Scanning Electron Microscope with X-Ray dispersion (SEM-EDX) to know cellulose and cellulose acetate morphology and chemical composition of EFB and DJL comparing the results between cellulose and cellulose acetate from both materials. The methodology used to prepare and synthesize cellulose acetate are similar to what Anderen did in previous experimentation.

\footnotetext{
* Corresponding author: detris@,che.ui.ac.id, andersen.yunan@,gmail.com
} 


\section{Experimental}

\subsection{Materials}

The raw materials used in this study were empty fruit bunches obtained from puspitek complex, BSD city, Indonesia and dried jackfruit leaves from Faculty of Engineering, University of Indonesia, Depok, Indonesia.

\subsection{Preparation of Raw Materials}

Preparation for both raw materials is done simultaneously in the same way. EFB and DJL are cleaned from the remains of the still-sticking fruit and other impurities. Then EFB and DJL was washed with water and dried in the sun to dry. EFB and DJL fibers are separated and cut to a size of 1-2 $\mathrm{cm}$ then stored at room temperature before proceeding for the research phase

\subsection{Cellulose Extraction}

Cellulose extraction is a pure cellulose removal step from EFB and DJL by removing other constituent parts such as lignin, hemicellulose, protein, and fat by using alkali treatment method. Cellulose extraction from EFB and DJL has the same stages. Cellulose extraction consists of 2 stages, such as:

\subsubsection{Delignification with sodium hydroxide}

The prepared raw materials, according to previous study that produces maximum yield of cellulose incorporated in a $12 \%$ EFB mixture and DJL $10 \%$ with a volume of 30 times the weight of the raw material and heated for 3 hours at a temperature of $90^{\circ} \mathrm{C}-100^{\circ} \mathrm{C}$. The result of heating is then filtered and washed until the filtrate is neutral.

\subsubsection{Bleaching with peroxide}

The samples were then bleached with $10 \% \mathrm{H}_{2} \mathrm{O}_{2}$ with 20 times by weight of raw material for 2 hours. After that the sample was filtered and washed again until neutral and heated at $105^{\circ} \mathrm{C}$ temperature for 6 hours before stored.

\subsection{Cellulose Acetate Synthesis}

At this stage, the reactants used are cellulose, glacial acetic acid, and anhydrous acetic acid. The preparation of cellulose acetate from EFB and DJL has the same stages, such as :

\subsubsection{Activation}

The obtained cellulose was added with glacial acetic acid with a volume ratio of $1: 10$, and heated with hot plate strirer at $38^{\circ} \mathrm{C}$ for 60 minutes before $98 \%$ sulfuric acid catalyst was added with a ratio of 1 gram of cellulose using $0.4 \mathrm{ml}$ and reheated at a fixed temperature for 45 minutes.

\subsubsection{Acetylation}

The result of the activation process was added with anhydrous and glacial acetic acid with a ratio of $3: 2$. The volume ratio between cellulose and glacial acetic acid was made for EFB 1:10 and DJL 1: 5 $(w / v)$. The solution was then heated at $38^{\circ} \mathrm{C}$ for 30 minutes.

\subsubsection{Hydrolisis}

The process of hydrolysis is done by stopping the acetylation process first, then added aquades and glacial acetic acid with a ratio of 1:2 and heated at a temperature of $50^{\circ} \mathrm{C}$ for 30 minutes.

\subsubsection{Drying}

The drying was carried out in an oven at $105^{\circ} \mathrm{C}$ for 6 hours after the resulting cellulose acetate washed until neutral $\mathrm{pH}$ and acetate odor were gone. After drying is complete then we get the dry cellulose acetate in the form of crude fiber. The result of cellulose acetate drying will then be tested for its physical characteristics.

\subsection{Advanced Characteristic Tests}

Advanced characteristic tests for this study were done by conducting Fourier Transformation Infra Red (FTIR) and Scanning Electron Microscope with $\mathrm{X}$-Ray dispersion (SEM-EDX) as follows :

\subsubsection{Fourier Transformation Infra Red}

The FTIR analysis serves to obtain a comparison of the characterization of cellulose fibers and cellulose acetate as well as analysis of the functional groups of both. In molecular clusters the characteristics of FTIR can be used to detect specific groups in a particular range of waves. The samples to be analyzed with FTIR are cellulose and cellulose acetate from EFB and DJL. The FTIR test was conducted at the Chemical Processing Basic Laboratory located at the Department of Chemical Engineering, Faculty of Engineering, University of Indonesia. 


\subsubsection{Scanning Electron Microscope with X- Ray dispersion}

The SEM-EDX analysis is an analysis that aims to determine the condition of the sample consisting :

1. Topography: Analysis of surface and texture of materials

2. Morphology: Analysis of sample shape and size

3. Compositions : Analysis of the composition of the sample quantitatively and qualitatively

\section{Results and Discussion}

\subsection{Fourier Transformation Infra Red}

\subsubsection{Empty Fruit Bunches (EFB) results}

In Figure 1, the EFB cellulose is a functional group of $\mathrm{O}-\mathrm{H}$ in the absorption area of $3337 \mathrm{~cm}^{-1}$, the C-O functional group at the $1035 \mathrm{~cm}^{-1}$ wave number and the $\mathrm{C}-\mathrm{H}$ functional group at the $2910 \mathrm{~cm}^{-1}$ wave number comprising the hydrocarbon. The $\mathrm{C}=\mathrm{O}$ functional groups within the $1700-1750 \mathrm{~cm}^{-1}$ wave range have not been seen in the EFB cellulose. However, in EFB cellulose acetate there are four functional groups, ie the $\mathrm{O}-\mathrm{H}$ functional group at $3335 \mathrm{~cm}^{-1}$ of the wave number is perfectly acetylated because it has a high percentage of transmittance compared to the cellulose phase. The
4. Crystallographic information, ie the grain deposits on the material being observed.

The samples to be analyzed by SEM-EDX are cellulose and cellulose acetate samples from TKKS and DNK. The tests were conducted at the Center for Materials Processing and Failure Analysis (CMPFA) Laboratory at the Department of Metallurgical and Materials Engineering University of Indonesia level of depth in this study is better than that of Bahmid [2] and resembles the results of research by Dian [3]. The obvious difference is seen in the functional group $\mathrm{C}=\mathrm{O}$ at the wave number $1721 \mathrm{~cm}^{-}$ ${ }^{1}$ which becomes the cellulose differentiator of EFB against EFB cellulose acetate. This shows that the functional group of $\mathrm{O}-\mathrm{H}$ in cellulose has been substituted by the acetyl group to give the functional group $\mathrm{C}=\mathrm{O}$. The presence of four functional groups of $\mathrm{O}-\mathrm{H}, \mathrm{C}-\mathrm{O}, \mathrm{C}-\mathrm{H}$ and $\mathrm{C}=\mathrm{O}$ indicates that the successful EFB cellulose acetate has been formed in this study.

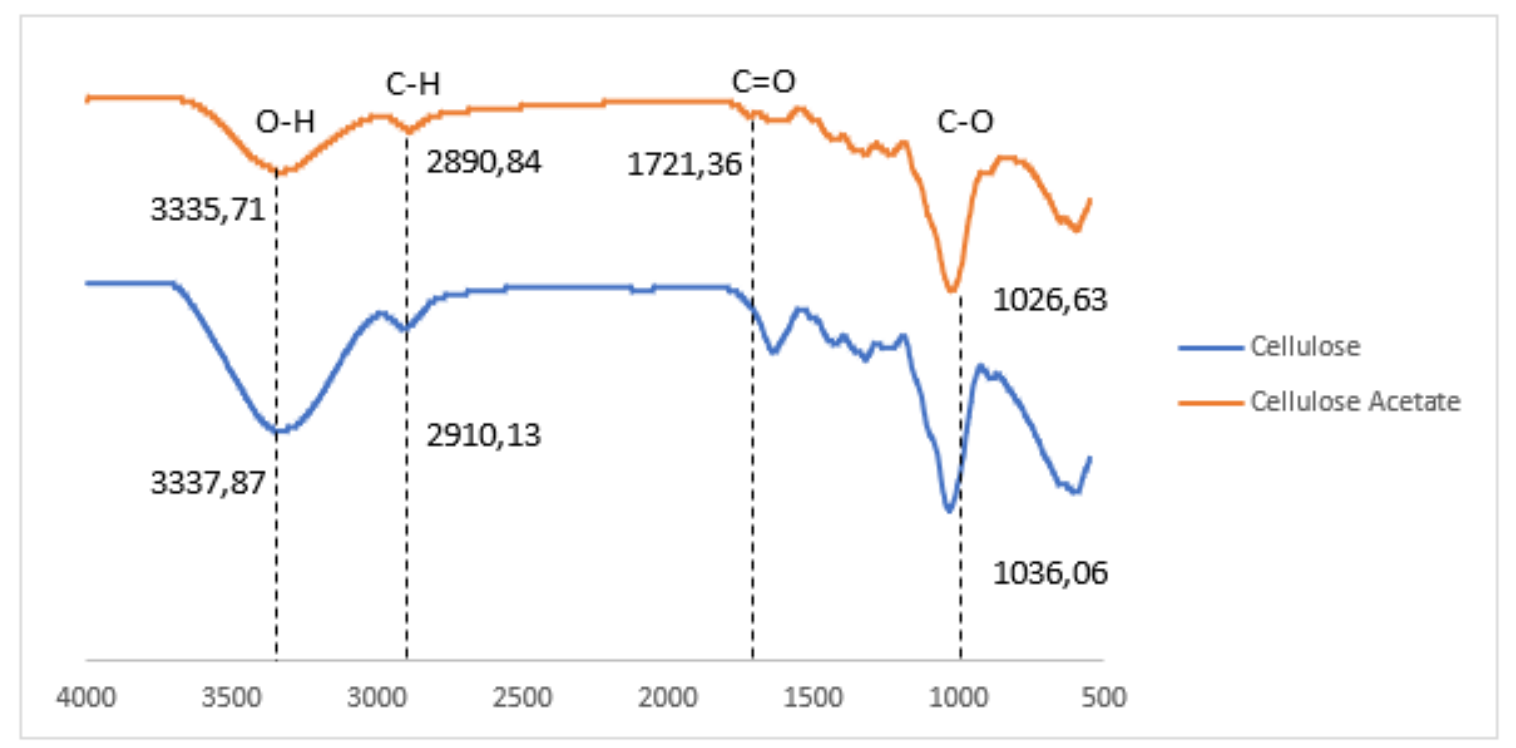

Fig 1. Graph of FTIR cellulose and cellulose acetate EFB analysis

\subsubsection{Dried Jackfruit Leaves Results}

Cellulose DJL and cellulose acetate DJL FTIR analysis shown in Figure 2 shows the presence of $\mathrm{OH}$ functional groups in the absorption area of 3338 $\mathrm{cm}^{-1}$, the $\mathrm{C}-\mathrm{O}$ functional group in the absorption region of $1043 \mathrm{~cm}^{-1}$ and the $\mathrm{C}-\mathrm{H}$ functional group at the wave number $2925 \mathrm{~cm}^{-1}$ for cellulose DJL. DJL cellulose acetate in Figure 2 shows a change in functional groups in the DJL cellulose acetate spectrum compared to DJL cellulose. The difference is the formation of $\mathrm{C}=\mathrm{O}$ carbonyl groups at a 
wavelength of $1725 \mathrm{~cm}^{-1}$. FTIR analysis of the spectrum proves that DNK cellulose acetate synthesis has been successfully performed. Also seen in Figure 2 that the wave number in the $\mathrm{O}-\mathrm{H}$ functional group has an increased percentage of transmittance compared to the DJL cellulose phase. cellulose acetate in which the synthesis of cellulose acetate has been perfectly formed. In comparison to cellulose acetate commercial and [4] results, the FTIR result between EFB and DJL show similarities to previous research. Hence, concluded cellulose acetate synthesis.

This shows the same characteristics of EFB

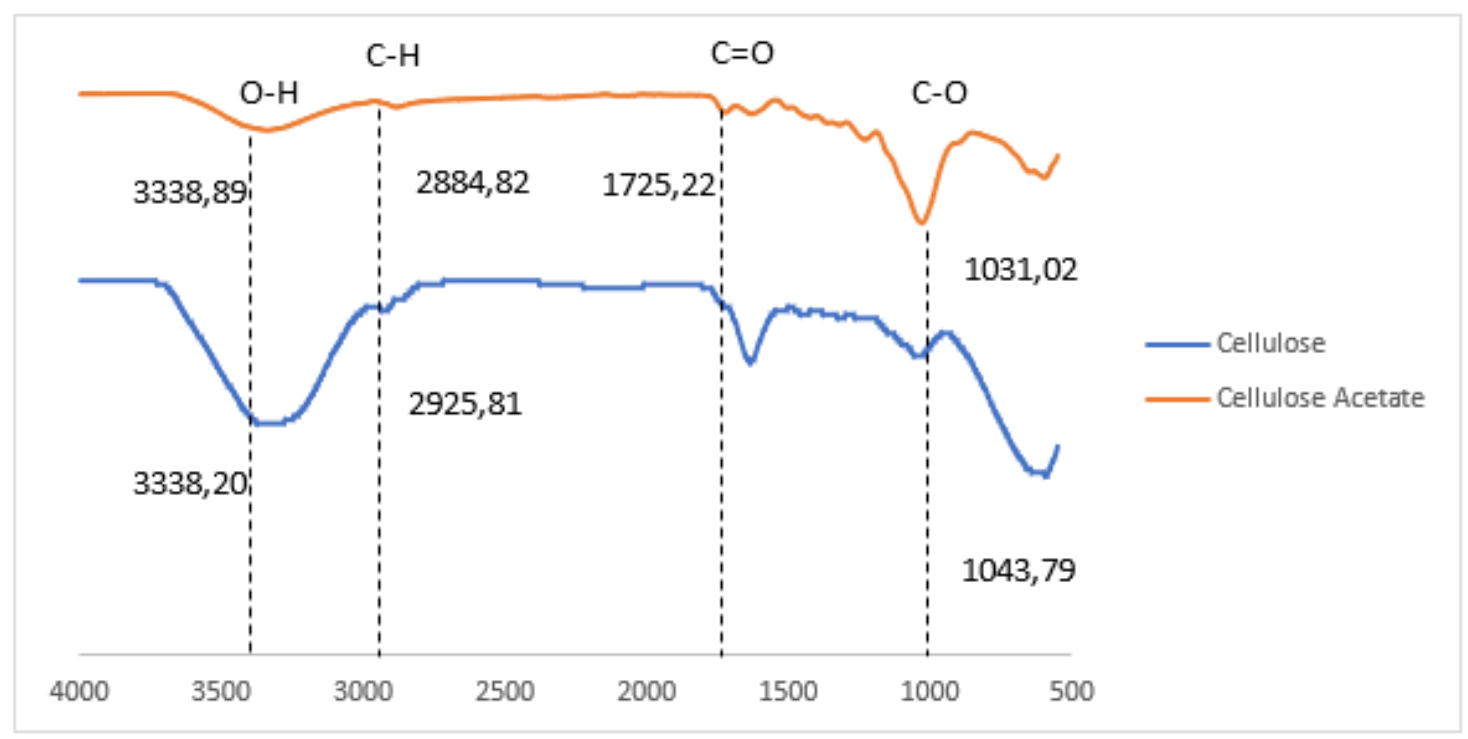

Fig 2. Graph of FTIR cellulose and cellulose acetate DJL analysis

\subsection{Scanning Electron Microscope with X-Ray dispersion}

\subsubsection{Empty Fruit Bunches (EFB) results}

The SEM-EDX analysis of the experimental cellulose acetate EFB (Figure $3 \mathrm{a}$ and $3 \mathrm{~b}$ ) shows the morphology of the homogeneous cylindrical cellulose acrylic powders that coalesce with the porous holes on its surface. The alteration of the porous layer on the surface of cellulose acetate is due to the acetylation process in cellulose to acetic anhydride. The obvious difference is seen when the EFB cellulose acetate is compared to the EFB cellulose which is irregularly shaped in the absence of small holes on its surface. The morphology of EFB cellulose acetate produced in this study differs somewhat from the results obtained by Bahmid [1], ie cellulose acetate produced in the form of a fibershaped cylinder and having small holes evenly distributed on its surface. Differences in pore distribution may be due to differences in cellulosic acetylation variation but in shape resemblance to the results of research by Bahmid [2] and Dian [3] although the shape and size of cellulose acetate produce within this experimentation are different from those of Bahmid and Dian because different source of which the raw materials were taken. The presence of white matter on the result of EFB cellulose acetate may be caused by impurities left during experimentation.

In the EDX analysis performed by X-ray diffraction on cellulose acetate to determine the composition of cellulose acetate obtained in Figure 4 where the composition of the produced EFB cellulose acetate proved to consist of carbon (C) and oxygen $(\mathrm{O})$. The composition of the hydrogen $(\mathrm{H})$ element which is supposed to be present in cellulose acetate does not come out in the EDX analysis results due to the low energy of hydrogen which can not be removed when emitted by X-rays. This proves that pure cellulose acetate is produced in the form of organic compounds and environmentally friendly. The results of the EDX analysis show that the produced EFB cellulose acetate is an organic compound in accordance with the chemical compound formula of cellulose acetate. 


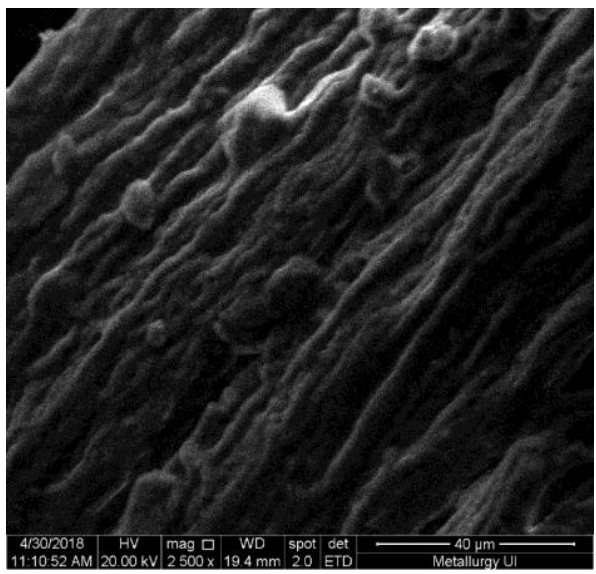

Fig 3a. SEM analysis of EFB Cellulose

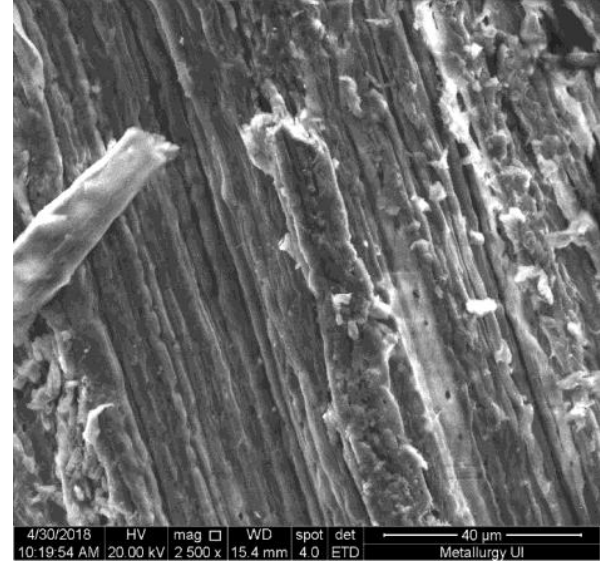

Fig $3 b$. SEM analysis of EFB cellulose acetate

\subsubsection{Dried Jackfruit Leaves Results}

The results of comparison of DJL cellulose analysis and DJL cellulose acetate powder in Figure 5a and $5 b$ show that the morphology of DJL cellulose acetate in the form of cylinder almost resembles the EFB cellulose acetate but the difference is seen from the asymmetrical cylinder form. Cellulose DJL has an irregular fiber shape in the absence of pores between fibers while DJL cellulose acetate has a fragile cylinder shape due to an asymmetrical shape. Comparison of EFB cellulose acetate in Figure 5 and DJL cellulose acetate shows that TKKS cellulose acetate is more robust due to a more symmetrical structure than DJL cellulose acetate. In DJL cellulose acetate, there are also small holes caused by acetylation processes. The presence of white matter on DJL cellulose acetate may be caused by impurities. The typical morphological differences in cellulose acetate EFB and DJL can be

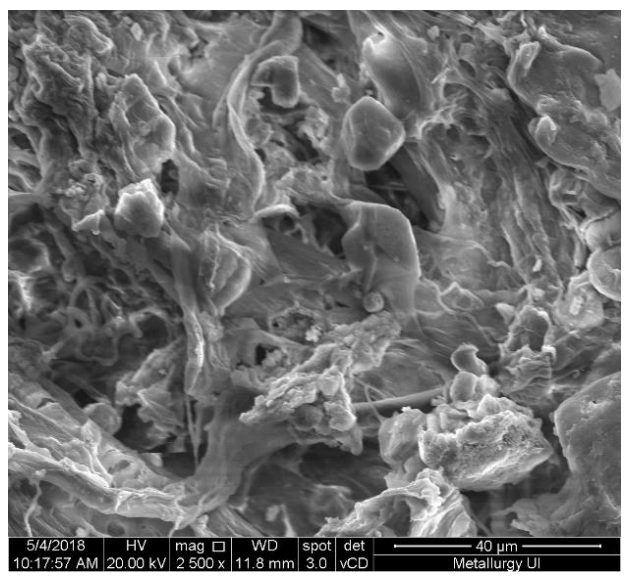

Fig 5a. SEM analysis of DJL cellulose caused by different types of cellulose sources. In addition, according to Vallejos [5] there is no change in the shape or morphology of cellulose acetate because there is an acetylated reaction, there is only the attachment of an acetyl group to the cellulose polymer molecule and does not alter the morphology.

The EDX analysis of DJL cellulose acetate in shows that the compositional composition of DJL cellulose acetate is an organic composition resembling EFB cellulose acetate, ie carbon (C) and oxygen $(\mathrm{O})$ in the absence of a hydrogen $(\mathrm{H})$ composition seen as a result of hydrogen energy is emitted on X-rays. It appears that a small inorganic silica ( $\mathrm{Si}$ ) composition on EDX analysis can be caused by impurities when tested because of the absence of $\mathrm{Si}$ compounds used when the study is either extracted or synthesized.

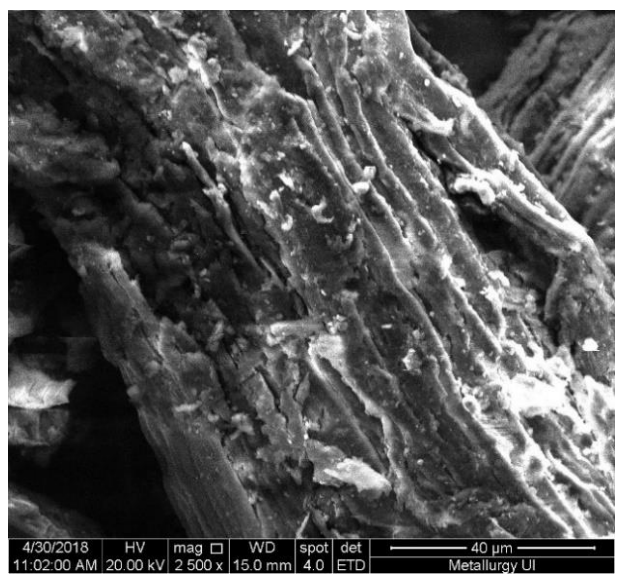

Fig 5b. SEM analysis of DJL cellulose acetate 
Table 1. EDX results of EFB cellulose acetate.

\begin{tabular}{|l|l|l|}
\hline Element & Wt\% & At\% \\
\hline CK & 46.27 & 53.42 \\
OK & 53.73 & 46.58 \\
Matrix & Correction & ZAF \\
\hline
\end{tabular}

Table 2. EDX results of DJL cellulose acetate.

\begin{tabular}{|l|l|l|}
\hline Element & Wt\% & At\% \\
\hline CK & 48.24 & 55.75 \\
OK & 50.01 & 43.38 \\
SiK & 01.75 & 00.87 \\
Matrix & Correction & ZAF \\
\hline
\end{tabular}

Table 1 and Table 2 showed the micronalaysis report of EDX for chemical compositions from cellulose acetate EFB and DJL in quantitavie results. Quantitative data between cellulose acetate EFB and DJL can only be compared with cellulose acetate chemical formula and has positive results in terms of atoms comparison.

\section{Conclusion}

In conclusion with FTIR testing, the typical uptake of TKKS and DNK cellulose acetates is produced by $\mathrm{C}=\mathrm{O}$ groups for $\mathrm{EFB}$ at wavelengths of 1721.36 $\mathrm{cm}^{-1}$ and DJL at wavelengths of $1725.22 \mathrm{~cm}^{-1}$. Meanwhile, in SEM-EDX testing, morphology and chemical composition of EFB and DJL cellulose acetate were obtained whereas EFB cellulose acetate was symmetrically cylindrical with small pores on its surface. DJL cellulose acetate has asymmetrical cylindrical shape with rare small pores on its surface. The chemical components of both cellulose acetate exhibit organic elements of carbon (C) and oxygen $(\mathrm{O})$ so that it can be concluded that the alternate alternative of these two raw materials is environmentally friendly.

\section{Acknowledgement}

This work was supported by PITTA grant from Universitas Indonesia.

\section{References}

1. D. Tristantini, Andersen. Characterization of cellulose acetate based on empty fruit bunches and dried jackfruit leaves as replacement candidates for microbeads. (to be published)
2. Bahmid, N. A., Khaswar S., Akhiruddin M., Pengaruh ukuran serat selulosa asetat dan penambahan dietilen glikol terhadap sifat fisik dan mekanik bioplastik, Jurnal Teknologi Industri Pertanian, 3, 226-234 (2014)

3. D. Tristantini, D.P. Dewanti, and C. Sandra, Isolation and characterization of $\alpha$-cellulose from blank bunches of palm oil and dry jackfruit leaves with alkaline process $\mathrm{NaOH}$ continued with bleaching process of $\mathrm{H}_{2} \mathrm{O}_{2}$ (2017)

4. Nurhayati, R. Kusumawati. Cellulose acetate synthesis from agar processing waste. $J P B$ Perikanan. E 9. 97-107 (2014)

5. Vallejos, M.e., Ppresin, M.S., \& Rojas, O.J. Allcellulose composite fibers obtained by electrospinning dispersions of cellulose acetate and cellulose nanocrystals. 5-6. (2012). 